\title{
Does hospitalization of a patient in the intensive care unit cause anxiety and does restriction of visiting cause depression for the relatives of these patients during COVID-19 pandemic?
}

\author{
Behiye Kosovali ${ }^{1}$, Nevzat Mehmet Mutluํ․ Canan Cam Gonen ${ }^{1}$, Tulay Tuncer Peker ${ }^{1}$, \\ Asiye Yavuz ${ }^{1}$, Ozlem Balkiz Soyal ${ }^{1}$, Esra Cakir ${ }^{1}$, Belgin Akan ${ }^{1}$, Derya Gokcinar ${ }^{1}$, Deniz \\ Erdem $^{1}$, and Isil Ozkocak Turan ${ }^{1}$ \\ ${ }^{1}$ Ankara City Hospital
}

February 24, 2021

\begin{abstract}
Objectives: During the pandemic, anxiety, stress, and depression may occur increasingly in the whole society. To evaluate the possible cause, incidence and levels of anxiety and depression in the relatives of the patients in the ICU in accordance with the patients' SARS-CoV-2 PCR result. Materials and Method: The study was prospectively conducted on relatives of patients admitted to tertiary ICU during COVID-19 pandemic. Sociodemographic characteristics of the patients and their relatives were recorded. "The Turkish version of the Hospital Anxiety and Depression Scale" questionnaire was applied twice to the relatives of 120 patients to determine the symptoms of anxiety and depression in accordance with the PCR results of the patients (PCR positive $\mathrm{n}=60$, PCR negative $\mathrm{n}=60$ ). Results: The ratios above cut-off values for anxiety and depression among relatives of the patients were $45,8 \%$ and $67,5 \%$ for the first test and $46,7 \%$ and $62,5 \%$ for the second test respectively. The anxiety and depression in the relatives of PCR positive patients was more frequent than the PCR negative ( $\mathrm{p}<0,001$ for HADS-A and $\mathrm{p}=0,034$ for HADS-D). The prevalence of anxiety and depression was significantly higher in female participants ( $\mathrm{p}=0,046$ for HADS-A and $\mathrm{p}=0,009$ for HADS-A). There was no significant correlation between HADS and age of the patient or education of the participants. The fact that the patients were hospitalized in the ICU during the pandemic was an independent risk factor for anxiety $(\mathrm{AUC}=0.746)$ while restriction of patient visiting in the ICU was found to be an independent risk factor for depression ( $\mathrm{AUC}=0.703)$. Conclusion: While patient with positive PCR and participant with female gender is responsible for both anxiety and depression, hospitalization in the ICU due to COVID-19 is an independent risk factor for anxiety and restriction of patient visiting in the ICU is an independent risk factor for depression.
\end{abstract}

Does hospitalization of a patient in the intensive care unit cause anxiety and does restriction of visiting cause depression for the relatives of these patients during COVID-19 pandemic?

\section{Abstract:}

Objectives: During the pandemic, anxiety, stress, and depression may occur increasingly in the whole society. To evaluate the possible cause, incidence and levels of anxiety and depression in the relatives of the patients in the ICU in accordance with the patients' SARS-CoV-2 PCR result.

Materials and Method: The study was prospectively conducted on relatives of patients admitted to tertiary intensive care units during COVID-19 pandemic. Sociodemographic characteristics of the patients and their relatives were recorded. "The Turkish version of the Hospital Anxiety and Depression Scale" questionnaire was applied twice to the relatives of 120 patients to determine the symptoms of anxiety and depression in accordance with the PCR results of the patients (PCR positive $n=60$, PCR negative $n=60$ ). 
Results: The ratios above cut-off values for anxiety and depression among relatives of the patients were $45.8 \%$ and $67.5 \%$ for the first test and $46.7 \%$ and $62.5 \%$ for the second test respectively. The anxiety and depression in the relatives of PCR positive patients was more frequent than the PCR negative $(\mathrm{p}<0.001$ for HADS-A and $p=0.034$ for HADS-D). The prevalence of anxiety and depression was significantly higher in female participants ( $\mathrm{p}=0.046$ for HADS-A and $\mathrm{p}=0.009$ for HADS-A). There was no significant correlation between HADS and age of the patient or education of the participants. The fact that the patients were hospitalized in the ICU during the pandemic was an independent risk factor for anxiety (AUC $=0.746$ ) while restriction of patient visiting in the ICU was found to be an independent risk factor for depression $(\mathrm{AUC}=0.703)$.

Conclusion: While patient with positive PCR and participant with female gender is responsible for both anxiety and depression, hospitalization in the ICU due to COVID-19 is an independent risk factor for anxiety and restriction of patient visiting in the ICU is an independent risk factor for depression.

Keywords: Anxiety, depression, hospital anxiety depression scale, intensive care unit, COVID-19, family members

\section{What's known?}

- Anxiety and depression levels of individuals increased in COVID-19 pandemic.

- The high rate of COVID-19 transmission worries the whole world.

- Worries are increasing for various reasons.

\section{What's new?}

We determined some of the causes of anxiety and depression in the relatives of patients treated ICU due to the COVID-19 in pandemic.

A relative is in the intensive care unit due to COVID-19 is an independent risk factor for anxiety and the restriction of visits in the ICU is an independent risk factor for depression.

\section{1 | INTRODUCTION:}

COVID-19 disease caused by SARS-CoV-2 virus, which was first isolated in Wuhan, China in December 2019, spread all over the world and caused a pandemic in a short time. The pandemic and its consequences still cause serious problems affecting people all over the world.

The answers to many questions such as how long the pandemic will last and when it will end, whether the vaccine will be effective are not yet clear. These uncertainties added to restrictions applied due to the epidemic also negate the socioeconomic status. In addition to all these, when COVID-19 is suspected in their loved ones, even otherwise healthy people may show symptoms of sleeping problems, stress, anxiety, and depression along with behaviors such as fear, anger, and denial. ${ }^{1}$

In Turkey, after the first case detected on 10th March 2020, despite intermittent quarantine practices and social isolation, COVID-19 cases persist and even rise. ${ }^{2}$ While some of these patients were treated at home, some were treated in hospital wards and intensive care units (ICU). Due to the high risk of human-to-human transmission of the SARS-CoV-2 virus, infected individuals must isolate themselves from the society as well as the family members throughout their illness. In our country, The Ministry of Health restricted patient visits, especially in the pandemic intensive care units starting from the beginning of the pandemic for the same reason. Relatives of these patients cannot see, touch, or talk to them under these circumstances. Another difference from the pre-pandemic period is that doctors report their patients' progress through phone calls, not face-to-face.

Anxiety and depression are not rare among relatives of the patients in the ICU. ${ }^{3}$ During the pandemic, visit and interview restrictions are expected to increase these symptoms. Even without a pandemic, we can say that being a relative of an intensive care patient is a stress factor itself while it is much worse in a pandemic which has many uncertainties. 
Various studies have been conducted to measure the degree of anxiety and depression caused by the pandemic and quarantine period in various patient groups or healthy individuals since the beginning of the pandemic. ${ }^{4,5}$ Post-traumatic stress disorder, anxiety and depression levels in general population are shown to be higher in China, where the pandemic started and then Italy, which is one of the most affected European countries, respectively. ${ }^{6,7}$ We did not encounter any study in the literature concerning anxiety and depression levels in the relatives of the patient being treated in the ICU due to COVID-19.

The aim of this study is to evaluate the anxiety and depression levels, incidences and the reasons causing them in the relatives of the patients treated in the ICU with positive or negative SARS-CoV-2 test results.

\section{2 | MATERIAL AND METHODS:}

\section{1 | Patient Data}

Our study was prospectively conducted on the relatives of the patients who were admitted to the tertiary pandemic intensive care units of Ankara City Hospital between May 15th and July 15th, 2020 after the approval of the ethics committee (Ethics committee number: E1-20-526).

This study included the native Turkish speaking relatives aged 18 and over of 120 patients with clinical or radiological suspicion of COVID-19 on admission to ICU, half of whom received positive PCR results $(\mathrm{n}=60)$ while the other half got negative PCR results $(n=60)$. The consents of the participants were obtained verbally during the phone call, due to the pandemic and the Ministry of Health's restriction of the hospital visits of the patient's relatives. The "Hospital Anxiety and Depression Scale (HADS)" questionnaire was applied to the relative of the ICU patient twice on phone by the intensive care doctor who followed the patient and gave information. Before HADS questionnaire, 7 different questions with 4-point scale ranging from 0 to 3 were asked to the participants in order to determine the causes of anxiety and depression (Table 1). HADS was first applied while the diagnosis of COVID-19 was not yet clear, and then repeated when the PCR test results were confirmed as positive or negative. Participants with previous or ongoing psychiatric illness as well as the ones who refused to participate in the study or cannot communicate and cooperate enough to complete the questionnaires during phone call were excluded from the study. Patients without confirmed PCR results were also excluded.

Gender, age, education (primary school, high school, university, illiterate), marital status (married, single, divorced, widow) of the patients and the participants were recorded. The patients were divided into two groups according to the Acute Physiology and Chronic Health Evaluation II (APACHE- II) score of the patient in the ICU as low mortality risk ([?]20) and high mortality risk ([?]21) groups. Expected mortality risks and PCR results were told to the participants on the phone, before the second questionnaire was applied. According to World Health Organization age classification, patients were divided into three groups as 18-65 years, 66-80 years, $81-99$ years. ${ }^{8}$ The degree of kinship was evaluated in four groups including spouse, child, relative or sibling. The occupations of the participants were classified as private employee, civil servant, unemployed or student.

HADS questionnaire, found by Zigmond and Snaith and translated and validated for the Turkish society by Aydemir et.al. was used to evaluate the degree of anxiety and depression of the participants. ${ }^{9,10}$ HADS consists of 14 questions with a 4-point scale ranging between 0-3 points. The general HADS score is the total score of all the 14 questions asked (0-42 points) while anxiety score (HADS-A) is calculated by adding up the 7 odd-numbered questions (0-21 points) and the depression score (HADS-D) by adding up the 7 even-numbered questions (0-21 points). The HADS questionnaire was administered to the participants on the phone and the score was calculated using the answers recorded. According to the validation of the HADS survey for the Turkish society, the values $>10$ and $>7$ were considered as cut-off values for anxiety and depression respectively (10). Participants were evaluated in 3 subgroups for anxiety and depression as normal (0-10 for HADS-A and 0-7 for HADS-D), moderate (11-15 for HADS-A and 8-10 for HADS-D) and high (16-21 for HADS-A and 11-21 for HADS-D).

\section{2 | Statistical analysis:}


Statistical analysis of the data obtained in the study was evaluated using the "SPSS for windows 23.0" statistical software. Continuous variables were expressed as mean \pm SD. After evaluating the conformity of numerical data to normal distribution by Shapiro-Wilkins test, student's t test was used to compare numerical data with normal distribution and the result was evaluated according to the equality of variances and MannWhitney U test was used to compare numerical data without normal distribution. Categorical data was given as numbers. Pearson Chi-Square test and Fisher Exact test were used to compare categorical data. Paired t or Wilcoxon Rank test was used after evaluating the compatibility of the difference to normal distribution in the comparison of the first and second survey results. Logistic regression analysis was performed to determine the factors associated with the presence of depression and anxiety. ROC curves were drawn for effective factors and the area under the curve (AUC) values were calculated. $<0.05$ was considered significant.

Post hoc power analysis of the study was calculated with G-Power, the power of the study was $86 \%$ for the comparison made according to the PCR result, $85 \%$ for the comparison made according to the participant's gender, $81 \%$ for the comparison made according to the APACHE-II score and the questions 1 and 3 obtained through logistic regression $88 \%$ and $82 \%$ respectively.

\section{3 | RESULTS:}

The results of 120 out of 160 patients' relatives were included in the statistical evaluation. Twenty two of the participants were excluded because their patients were transferred to the ward from the ICU, before the second test, 10 were excluded because their patients died before the second test was performed, 7 were excluded because of unavailability through phone, and 1 participant was excluded because the patient was coming from a nursing home and the survey was taken by a staff, not a relative.

When all patients evaluated, the average age was 70.22. Sixty (50\%) patients were male with an average age of 66.48 and sixty (50\%) were female with an average age of 73.95 . Ninety two $(76.7 \%)$ of the patients were married and $72(60 \%)$ of them were graduated from the primary school. The average APACHE-II score was 17 and $34(28 \%)$ patients required mechanical ventilator (Table 2).

When all participants were evaluated, the average age was $43.88 .72(60 \%)$ were male, $94(78.3 \%)$ were married, 54 (45\%) were graduated from the university, $83(69.2 \%)$ were children of the patients, $53(44.2 \%)$ were private employee (Table 3 ).

While there was no difference in the averages of HADS, HADS-A and HADS-D between the first and second surveys $(\mathrm{p}=0.572, \mathrm{p}=0.974, \mathrm{p}=0.190$ respectively). Participants with HADS-A and HADS-D anxiety and depression scale above the cut-off values were $45.8 \%$ and $67.5 \%$ for the first test and $46.7 \%$ and $62.5 \%$ for the second test respectively (Table 4).

Although there was no statistically significant difference between the averages of the first questionnaire according to the PCR results of the patients $(\mathrm{p}=0.315)$, and the ratio of participants with PCR positive patients who have higher survey scores then the cut-off values for anxiety and depression were higher than the PCR negative patients both in HADS-A ( $51.6 \%$ for PCR positive, $\mathrm{n}=31$ and $40 \%$ for PCR negative, $\mathrm{n}=24$ ) and HADS-D ( $70 \%$ for PCR positive, $\mathrm{n}=41$ and $65 \%$ for PCR negative, $\mathrm{n}=39$ ) for the first questionnaire. When the results of the second questionnaire were evaluated, HADS, HADS-A and HADS-D averages were significantly higher $(\mathrm{p}=0.001, \mathrm{p}<0.001, \mathrm{p}=0.012$ respectively), also the ratio of participants with $\mathrm{PCR}$ positive patients who have higher survey scores then the cut-off values for anxiety and depression were significantly higher than the PCR negative patients ( $\mathrm{p}<0.001$ for HADS-A and $\mathrm{p}=0.034$ for HADS-D) (Table 5).

When compared according to gender, the HADS and HADS-A scores of the first questionnaire and the HADS-D scores of the first and second survey were significantly higher in female then male participants $(\mathrm{p}=0.014,0.046,0.009,0.049$ respectively) (Table 6).

When HADS results were compared according to kinship, the HADS and HADS-A results of the first questionnaire were significantly higher among spouses of the patients than the other relatives $(\mathrm{p}=0.05$ and $\mathrm{p}=0.020$ respectively) (Table 7 ). 
When the first and second questionnaire HADS results were compared in terms of APACHE-II score, there was no statistical difference ( $\mathrm{p}=0.919)$, but the HADS-D results of the second questionnaire were significantly higher for patients with an APACHE-II score [?]21 $(\mathrm{p}=0.042)$ (Table 8).

The average HADS values of participants did not change according to age of the patients, but the HADS average of the participants increased as the age of the patients decreased and, although not statistically significant, as the age of the patients increased, anxiety and depression scales of the participants decreased (Table 9).

No significant relation was found between the education of the participants and the HADS results (Table 10).

Logistic regression analysis was used to evaluate whether the answers to the questions asked to the participants were independent risk factors for anxiety and depression which showed patients' hospitalization in the intensive care unit due to pandemic to be an independent risk factor for anxiety among the participants while restrictions to visit patients in the intensive care unit to be an independent risk factor for depression (Table 11, Table 12). ROC curves were drawn. For anxiety in the participants, $\mathrm{AUC}=0.746$ for question 1 and for depression, $\mathrm{AUC}=0.703$ for question 3 (Figure 1, Figure 2).

Twenty five of the participants stated 10 different reasons for anxiety and depression. Five of them feared death of their patient, 4 feared infecting their families, 3 feared infecting other people, 3 feared the length of time to recovery, 3 feared loss of their jobs or had financial issues, 2 were upset about not getting convenient information through regular calls, 1 was anxious about the education of his child, 1 was anxious because he started working and could get infected, 1 expressed concern about the general spread of the disease and the increasing number of patients, while another expressed concern about the insufficiency and unreliability of the data announced by the Ministry of Health.

\section{4 | DISCUSSION:}

Although there aren't any studies in the literature evaluating the anxiety and depression in the relatives of the patients during the pandemic, it has been reported before the pandemic that being a relative of a hospitalized patient in the intensive care unit due to a life-threatening disease is an important stress factor and may cause anxiety and depression. ${ }^{11}$ Anxiety and depression rates of relatives of patients in the ICU were evaluated by studies conducted in different countries before the pandemic some of which are as follows: $69.1 \%$ and $35.4 \%$ in a multicenter trial in France, $71.8 \%$ and $53.8 \%$ in a trial reported from Brazil, $60 \%$ and $54 \%$ in another trial from Brazil, $35 \%$ and $66 \%$ in a trial from India, $35.9 \%$ and $71.8 \%$ in a trial from Turkey respectively. ${ }^{11,12,13,14,15}$ As a result of these trials preceding the pandemic, the anxiety levels in our country is lower than France and Brazil, similar to India while depression levels are higher than France, Brazil and India. When we compare the rates of anxiety and depression in relatives of the patients before the pandemic (35.9\% and $71.8 \%$ respectively) by Kose et al. with our study, we can roughly tell that anxiety levels rised $(45.8-46.7 \%)$ while depression levels decreased $(62.5-67.5 \%)$ during the pandemic. ${ }^{15}$

Some of the studies showing the rates of anxiety and depression on the general population during the pandemic are as follows: $29.83 \%$ and $16.76 \%$ in Russia, $35 \%$ and $22 \%$ in Austria, $28.8 \%$ and $16.5 \%$ in China, and $45.1 \%$ and $23.6 \%$ in Turkey respectively. ${ }^{16,17,18,19}$ In our country, the anxiety rates in the general population were lower than Russia and Austria but higher than China while depression rates were higher than the rest. Apart from cultural differences, the differences in health care between countries and the onset of the trials as the beginning or mid-pandemic period or the level of awareness may be responsible for the results. One of the reasons why the anxiety and depression rates in our study were higher than China may be because in China, the study was carried out by WHO as soon as the pandemic was declared while our study 3-6th months of the pandemic in our country. The official onset of the pandemic was different between countries as well. ${ }^{18}$ This later onset of COVID-19 pandemic in our country may be cause of higher rates of anxiety and depression because of the awareness of transmission routes and speed as well as the mortality rates. National and social media shares updated information about number of patients, deaths in the country and the world which inclines day by day may also be responsible for the anxiety and depression rates. Ozdin 
et al also conducted a study concerning the anxiety and depression among healthy volunteers during the pandemic. ${ }^{19}$ Depression rates of our study was higher probably because of the population we chose.

In the literature, there is no similar study conducted with relatives of patients hospitalized in ICU during pandemic. Our trial was started at the beginning of the pandemic and all patients were admitted to ICU with suspicion of COVID-19. The diagnosis was confirmed with PCR in addition to clinical and radiological findings. The higher rates of anxiety and depression in the relatives of patients with positive PCR test can be explained by the serious concerns about the disease. A positive PCR also eliminates the possibility of not having the disease and can incline anxiety and depression.

In our study we found that symptoms of anxiety and depression are more common in women during the pandemic, consistent with the studies prior to pandemic showing female susceptibility to anxiety and depression. ${ }^{20,21}$ Studies from different countries conducted in the general population since the beginning of the pandemic also show tendency to anxiety and depression in female gender. ${ }^{6,17,23,24}$ During the pandemic, female gender was emphasized with regard to anxiety and depression in our country as well. ${ }^{19}$ Hormonal changes during the menstrual cycle can cause mood changes, which can cause women's reactions to events to be more exaggerated or negative than men. ${ }^{25}$ Considering that women have more posttraumatic stress symptoms such as negative alteration of cognition and mood, re-experiencing and hyperarousal than men in the COVID-19 epidemic, they are expected to have more anxiety and depression symptoms due to both the pandemic and the anxiety they feel for the wellbeing of their patients. ${ }^{23}$ Another factor affecting depression is the role that societies attribute to genders depending on cultural differences. ${ }^{22}$ Although the study is carried out in the capital of our country, the cultural mosaic in the city can reflect almost every region of our country since it is a city that continues to receive immigrants from all over the country. In some societies, the under-reacting of women to the events is regarded as abnormal, while in some regions, the overreaction of men is abnormal. Due to the place of men in society and the role assigned to them, men can show their emotions less than women. The characteristics of the regions where people come from, where they grow up, family and economic structure may be factors that make the reactions of women and men different from each other. Cultural characteristics and the acceptance that the female gender may be emotionally reactive in life may explain the higher frequency of anxiety and depression among females.

Another factor in which anxiety and depression rates were significantly higher in our study was if the participant was the spouse of the patient. After the spouses, the children, relatives, and siblings of the patients followed the frequency of symptoms of anxiety and depression among the relatives. The result of our study was consistent with previous studies. ${ }^{15,27}$ Because the spouses share a house, a life, and values, one's illness of affects the surviving spouse both emotionally and socioeconomically. Therefore, we believe that it is an expected result that the symptoms of anxiety and depression are more common in spouses compared to other relatives of the ICU patients.

People aged 65 and over are more likely to have COVID-19 disease and especially respiratory failure and the need for intensive care than younger patients. However younger patients may also need treatment in intensive care and death of these patients is more devastating for their relatives. In our study, although the age of the patient did not significantly affect the anxiety and depression levels of their relatives, the rates of high anxiety and depression was higher in the relatives of young patients than those of middle and elderly patients. In this aspect, it was consistent with the results of other studies. ${ }^{13,26}$ In the literature, there is no difference between the anxiety and depression levels of the relatives of ICU patients in terms of scores predicting mortality or the education level of the patients' relatives. ${ }^{15,29,30} \mathrm{In}$ our study, we observed that learning the severity of the disease (high APACHE-II score) by the relatives of the patients did not increase the anxiety level, but it increased the depression. The higher incidence of depression among the relatives of the patients in the ICU can be because their patients are hospitalized in intensive care with the diagnosis of COVID-19, knowing that their condition is more severe and possibly lethal. Most of the participants in the study were university graduates, and although the lowest anxiety and depression levels were found in this group, and there was no significant relationship between the education level of the participants and their anxiety or depression levels. This suggests that education may be effective in the perception process 
and acceptance of results, but still cannot fully control emotional responses. Our study associates anxiety with COVID-19 as an independent risk factor in accordance with to the answer given to the question "How concerning is your patient's hospitalization in the ICU due to an epidemic?" by the participants. A relative hospitalized in the ICU due to an epidemic was found to be effective in the development of anxiety.

With the restrictions made to prevent transmission during the pandemic period and the prohibition of daily patient visits, patients' relatives could not visit their patients in the intensive care environment. Therefore, we think that the lack of seeing, communicating and physical contact in the ICU increases the curiosity and anxiety of the relatives of the patients. Not being able to visit and see a patient was found to be an independent risk factor for the development of depression in the participants.

There may be many different factors that can cause anxiety and depression on people during the pandemic period. Since we had only 25 participants who answered the question about other causes of anxiety and depression, these answers were not evaluated statistically. From these answers, which we have also stated in the findings section, we think that 10 different reasons may cause anxiety in the relatives of the patient during the pandemic process. We believe that these reasons should be questioned in future studies investigating the causes of anxiety and depression.

In conclusion, during the pandemic period, the fact that a relative is in the intensive care unit due to COVID-19 is an independent risk factor for anxiety and the restriction of visits in the ICU is an independent risk factor for depression.

Limitations: Single center, the number of participants, taking the questionnaire by phone are the limitations of our study.

\section{Author Contributions:}

Behiye Deniz Kosovali: Concept/design, Data analysis/interpretation, Data collection, Drafting article

Nevzat Mehmet Mutlu: Concept/design, Data collection

Canan Cam Gonen: Data collection

Tulay Tuncer Peker: Data analysis/interpretation, Statistics

Asiye Yavuz: Data collection

Ozlem Balkiz Soyal: Data analysis/interpretation, Drafting article

Esra Cakır: Data collection

Belgin Akan: Data collection

Derya Gokcinar: Data collection

Deniz Erdem: Data collection

Isıl Ozkocak Turan: Data collection

\section{REFERENCES:}

1. Torales J, O'Higgins M, Castaldelli-Maia J.M, Ventriglio A. The outbreak of COVID-19 coronavirus and its impact on global mental health.International Journal of Social Psychiatry. 2020;66:317-320. 


\section{Republic Of Turkey Ministry Covid-19 Information Pagehttps://covid19.saglik.gov.tr}

3. Kourti M, Christofilou E, Kallergis G. Anxiety and depression symptoms in family members of ICU patients. Av Enferm.2015;33:47-54.

4. Ernstsen L, Havnen A. Mental health and sleep disturbances in physically active adults during the COVID-19 lockdown in Norway: does change in physical activity level matter? Sleep Med . 2021;77:309312 .

5. Romito F, Dellino M, Loseto G, et. al. Psychological distress in outpatients with lymphoma during the COVID-19 pandemic. Front. Oncol. 2020;10:1270.

6. Wang C, Pan R, Wan X, Tan Y, Xu L, Ho CS, et al. Immediate psychological responses and associated factors during the initial stage of the 2019 coronavirus disease (COVID-19) epidemic among the general population in China. Int $J$ Environ Res Public Health.2020;17:E1729.

7. Rossi R, Socci V, Talevi D, Mensi S, Niolu C, Pacitti F, et al. COVID-19 pandemic and lockdown measures impact on mental health among the general population in Italy. An $\mathrm{N}=18147$ web-based 3 survey. Front Psychiatry. 2020;11:790.

8. https://www.who.int/health-topics/ageing

9. Zigmond A.S, Snaith R.P. The Hospital anxiety and depression scale.Acta psychiatr. scand. 1983:67:361370.

10. Aydemir Ö, Güvenir T, Küey L, Kültür S. Hastane anksiyete ve depresyon ölçeğinin ürkçe formunun geçerlilik ve güvenilirlik çalışması. Türk Psikiyatri Dergisi. 1997;8:280-287.

11. Pochard F, Elie Azoulay E, Chevret S et al. Symptoms of anxiety and depression in family members of intensive care unit patients: Ethical hypothesis regarding decision-making capacity. Crit Care Med.2001;29,10

12. Maruiti M.R , Galdeano L.E, Farah O.G.D. Anxiety and depressions in relatives of patients admitted in intensive care units. Acta Paul Enferm. 2008;21:636-42.

13. Midega T.D, Barros de Oliveira H.S, Fumis R.R.L. Satisfaction of family members of critically ill patients admitted to a public hospital intensive care unit and correlated factors. Rev Bras Ter Intensiva. 2019;31:147155 .

14. Borges R, Prasanth Y.M. Psychological and socio-economic burden on families of patients admitted in intensive care unit.International Journal of Biomedical Research. 2016;7:733-737.

15. Köse I, Zincircioğlu Ç, Öztürk Y.K, Factors affecting anxiety and depression symptoms in relatives of intensive care unit patients. Journal of Intensive Care Medicine ; 2016;3:611-7.

16. Karpenko O.A, Syunyakov T.S, Kulygina M.A, Impact of COVID-19 pandemic on anxiety, depression and distress - online survey results amid the pandemic in Russia. Consortium Psychiatricum. DOI: 10.17650/2712-7672-2020-1-1-8-20.

17. Fauziah Rabbani F, Khan H.A, Piryani S, Khan A.R, Abid F. Psychological and social impact of COVID19 in Pakistan: Need for gender responsive policies. doi: https://doi.org/10.1101/2020.10.28.20221069

18. Wang C ,Pan R ,Wan X et al. Immediate Psychological responses and associated factors during the initial stage of the 2019 coronavirus disease (COVID-19) epidemic among the general population in China.Int. J. Environ. Res. Public Health. 2020;17:1729. 
19. Özdin S, Özdin Ş.B. Levels and predictors of anxiety, depression and health anxiety during COVID19 pandemic in Turkish society: The importance of gender. International Journal of Social Psychiatry.2020;66,504-511.

20. Bastian Matt B, Schwarzkopf D, Reinhart K, König C, Hartog C.S. Relatives' perception of stressors and psychological outcomes - Results from a survey study. Journal of Critical Care. 2017;39:172-177

21. Kourti M, Christofilou E, Kallergis G. Anxiety and depression symptoms in family members of ICU patients. Av Enferm.2015;33:47-54.

22. Azoulay E, Cariou A, Bruneel F, et. al. Symptoms of anxiety, depression and peritraumatic dissociation in critical care clinicians managing COVID-19 patients: A Cross-sectional study. American Journal of Respiratory and Critical Care Medicine. 2020:202;1388-1398.

23. Liua N, Zhang F, We C, et al. Prevalence and predictors of PTSS during COVID-19 outbreak in China hardest-hit areas: Gender differences matter. Psychiatry Research . 2020;287:112-921.

24. Gerhold L. COVID-19: Risk perception and coping strategies. Results from a survey in Germany $10.31234 /$ osf.io/xmpk4.

25. Soni M, Curran V.H, Kamboj S.K. Identification of a narrow post-ovulatory window of vulnerability to distressing involuntary memories in healthy women. Neurobiology of Learning and Memory.2013; 104:32-38

26. Arthur Kleinman. Culture and Depression. N Engl J Med. 2004;351:951-3.

27. Siegel MD, Hayes E, Vanderwerker LC, Loseth DB, Prigerson HG. Psychiatric illness in the next of kin of patients who die in the intensive care unit. Crit Care Med . 2008;36:1722-1728.

28. McAdam J.L, Dracup K.A, White D.B, Fontaine D.K, Puntillo K.A, Symptom experiences of family members of intensive care unit patients at high risk for dying. Crit Care Med. 2010;38:1078-85

29. Delva D, Vanoost S, Bijttebier P, Lauwers P, Wilmer A. Needs and Feelings of Anxiety of Relatives of Patients Hospitalized in Intensive Care Units. Soc Work Health Care. 2002;35:21-40.

30. Rengin Acaroğlu, Hatice Kaya, Merdiye Şendir, Kezban Tosun, Yüksel Turan. Levels of anxiety and ways of coping of family members of patients hospitalized in the Neurosurgery Intensive Care Unit.Neurosciences (Riyadh). 2008;13:41-5.

\section{Hosted file}

tables.pdf available at https://authorea.com/users/397896/articles/510642-doeshospitalization-of-a-patient-in-the-intensive-care-unit-cause-anxiety-and-doesrestriction-of-visiting-cause-depression-for-the-relatives-of-these-patients-duringcovid-19-pandemic

\section{Hosted file}

figures.pdf available at https://authorea.com/users/397896/articles/510642-doeshospitalization-of-a-patient-in-the-intensive-care-unit-cause-anxiety-and-doesrestriction-of-visiting-cause-depression-for-the-relatives-of-these-patients-duringcovid-19-pandemic 